Aim: To evaluate clinical usefulness of metagenomics sequencing alongside conventional testing in two adult cases of CNS infection.

Methods: For both cases, bacterial microscopy and culture, herpes multiplex PCR, enterovirus PCR, parasitology multiplex PCR, Acanthamoeba culture, fungal culture, cryptococcal antigen and $M$. tuberculosis culture and PCR were done on the cerebrospinal fluid (CSF) with $4 \mathrm{~mL}$ required for each patient. Metagenomics sequencing required only $0.4 \mathrm{~mL}$ of CSF for each patient. CSF was subjected to particle enrichment (virus and ribosomes), random amplification of extracted RNA and DNA, library preparation and Ion Torrent next generation sequencing (NGS). Although clearly an experimental method, this method has been successful in detecting viruses and other microbes in a range of samples.

Result: All conventional testing as mentioned above was negative. NGS of sample 1 generated 3.7 million reads, mean read length 156 nucleotides; sample 2 generated 4 million reads, mean read length 185 nucleotides. Analysis of NGS reads focusing on finding an infectious aetiology, did not reveal any significant findings. Clinicians were reassured to prescribe immune-suppressive therapy with good response for Case 1 and have antibiotics ceased in Case 2 .

Conclusion: Metagenomics sequencing alongside conventional testing may be useful for ruling out active infection in patients with possible CNS infection.

\section{A CASE OF BILHARZIAL TUBERCLE IN A MIGRANT FROM ZIMBABWE, DIAGNOSED LATE THROUGH CYSTOSCOPY AND HISTOLOGY, WITH NEGATIVE SCHISTOSOMIASIS SEROLOGY [INDIRECT HEMAGGLUTINATION (IHA)]}

\author{
Kwee Chin Liew ${ }^{1}$, Carolyn Beckett \\ ${ }^{1}$ Department of Infectious Diseases, Eastern Health, Vic, \\ Ausralia
}

We report a case of Bilharzial tubercle in a migrant from Zimbabwe, recognised 10 years later through flexible cystoscopy and histological findings. A 21-year-old female presented to her local doctor with six months history of supra-pubic pain and dyspareunia without systemic symptoms. Her past medical history included intermittent asymptomatic haematuria since 2006 attributed to thin membrane disease, migraine and sinusitis. She had freshwater exposure in a rural region in Africa prior to migration to Australia 11 years ago. Her urine dipstick showed erythrocytes count of $137 \times 10^{6} / \mathrm{L}$. Schistosomiasis serology (IHA) was negative. Pelvic ultrasonography showed a focal $8 \times 5 \mathrm{~mm}$ lesion in the posterior bladder wall suspicious for neoplastic aetiology. Cystoscopy demonstrated patchy changes on bladder mucosa and a persistent bladder wall mass, which was nodular and suspicious for Bilharzial tubercle. A $7 \mathrm{~mm}$ fragment of the bladder lesion showed urothelium with mucosal ulceration and dense mixed inflammation with large numbers of eosinophils. Numerous calcified schistosoma eggs were present within the mucosa and lamina propria. The mucosa showed reactive changes and there was benign squamous metaplasia without evidence of malignancy. She was treated with praziquantel $20 \mathrm{mg} / \mathrm{kg}$ orally with food, for 2 doses and 4 hours apart with resolution of her symptoms, haematuria and lesion.

\section{EVALUATING COMMUTABILITY OF EQA SAMPLES FOR EPSTEIN-BARR VIRUS SEROLOGY}

\author{
Ben Limoux, Shabeena Ali, Grace Moyo, Farisha Firoz, \\ Peter Graham \\ The Royal College of Pathologists of Australasia Quality \\ Assurance Programs (RCPAQAP), St Leonards, Sydney, \\ Australia
}

Background: Providing 'patient-like' commutable samples is an ongoing challenge for all EQA providers. RCPAQAP sources human serum and plasma from commercial suppliers, donated de-identified pools and consenting donors. For some assays plasma is not the recommended specimen type for testing.

Aim: In 2019, RCPAQAP performed plasma to serum conversion for EBV surveys previously distributed as a plasma sample to investigate the effect of sample type on results.

Methods: A single source plasma sample (from Physicians Plasma Alliance collected in sodium citrate) was distributed for two EBV surveys. RCPAQAP subsequently performed a plasma to serum conversion using an in-house method and redistributed the defibrinated sample for a third survey. A comparison of the replicate samples was performed to identify any discrepancies.

Results: A shift in reporting for qualitative results (positive/ negative) for EBV VCA IgM was noted over the three surveys. The two surveys using plasma samples had less than $80 \%$ agreement across methods whereas a consensus of $90 \%$ was achieved for the defibrinated sample.

Discussion: There are many contributing factors that can lead to variation in results including sample type. For serological testing, it is important to be aware of any limitations for the assay in use. RCPAQAP now routinely defibrinates plasmas where required to improve the commutability of the sample.

\section{EVALUATING A STRONGYLOIDES EQA PROGRAM: OUTCOMES OF A TWO-YEAR PILOT STUDY}

Grace Moyo, Shabeena Ali, Farisha Firoz, Peter Graham

The Royal College of Pathologists of Australasia Quality

Assurance Programs (RCPAQAP), St Leonards, NSW, Australia

\begin{abstract}
Aim: While an initial Strongyloides pilot survey in 2018 showed a high concordance in reporting of qualitative results, there was a high variation in the reporting of quantitative results within and between method groups. In 2019, RCPAQAP Serology distributed a second Pilot Program to assess the reporting of low-level positive results.

Method: The 2019 survey (comprising two duplicate negative, one high-level and one low-level positive serum samples) was distributed to 12 Australian and 5 international laboratories for Strongyloides IgG and total antibody testing. Qualitative (pos/ neg) and quantitative (NTU, OD, and S/CO) data were subsequently analysed using RCPAQAP in-house software.

Results: 16 participants used a commercial assay and one an inhouse assay. Qualitative results showed $100 \%$ consensus for the negative specimens, $81 \%$ consensus for the high-level positive samples, and no consensus for the low-level positive sample. A high variation in the reporting of quantitative results between and within method groups and inconsistencies in the reporting of cutoff values were identified.
\end{abstract}

